\title{
BMJ Open Role of cardiac CT in the diagnostic evaluation and risk stratification of patients with myocardial infarction and non-obstructive coronary arteries (MINOCA): rationale and design of the MINOCA-GR study
}

Georgios P Rampidis (D) , ${ }^{1,2}$ Polydoros N Kampaktsis, ${ }^{3}$ Konstantinos Kouskouras, ${ }^{4}$ Athanasios Samaras, ${ }^{1}$ Georgios Benetos, ${ }^{5}$ Andreas A Giannopoulos, ${ }^{6}$ Theodoros Karamitsos, ${ }^{1}$ Alexandros Kallifatidis, ${ }^{7}$ Antonios Samaras, ${ }^{8}$ Ioannis Vogiatzis (i) , ${ }^{8}$ Stavros Hadjimiltiades, ${ }^{1}$ Antonios Ziakas, ${ }^{1}$ Ronny R Buechel, ${ }^{6}$ Catherine Gebhard, ${ }^{6}$ Nathaniel R Smilowitz, ${ }^{3}$ Konstantinos Toutouzas, ${ }^{5}$ Konstantinos Tsioufis, ${ }^{5}$ Panagiotis Prassopoulos, ${ }^{4}$ Haralambos Karvounis, ${ }^{1}$ Harmony Reynolds, ${ }^{9}$ George Giannakoulas (D) ${ }^{1}$

To cite: Rampidis GP, Kampaktsis PN, Kouskouras K, et al. Role of cardiac CT in the diagnostic evaluation and risk stratification of patients with myocardial infarction and non-obstructive coronary arteries (MINOCA): rationale and design of the MINOCA-GR study. BMJ Open 2022;12:e054698. doi:10.1136/ bmjopen-2021-054698

- Prepublication history for this paper is available online. To view these files, please visit the journal online (http://dx.doi. org/10.1136/bmjopen-2021054698).

Received 20 June 2021 Accepted 22 December 2021

Check for updates

(c) Author(s) (or their employer(s)) 2022. Re-use permitted under CC BY-NC. No commercial re-use. See rights and permissions. Published by BMJ.

For numbered affiliations see end of article.

Correspondence to Dr George Giannakoulas; ggiannakoulas@auth.gr

\section{ABSTRACT}

Introduction Myocardial infarction with non-obstructive coronary arteries (MINOCA) occurs in 5\%-15\% of all patients with acute myocardial infarction. Cardiac MR (CMR) and optical coherence tomography have been used to identify the underlying pathophysiological mechanism in MINOCA. The role of cardiac CT angiography (CCTA) in patients with MINOCA, however, has not been well studied so far. CCTA can be used to assess atherosclerotic plaque volume, vulnerable plaque characteristics as well as pericoronary fat tissue attenuation, which has not been yet studied in MINOCA.

Methods and analysis MINOCA-GR is a prospective, multicentre, observational cohort study based on a national registry that will use CCTA in combination with CMR and invasive coronary angiography (ICA) to evaluate the extent and characteristics of coronary atherosclerosis and its correlation with pericoronary fat attenuation in patients with MINOCA. A total of 60 consecutive adult patients across 4 participating study sites are expected to be enrolled. Following ICA and CMR, patients will undergo CCTA during index hospitalisation. The primary endpoints are quantification of extent and severity of coronary atherosclerosis, description of high-risk plaque features and attenuation profiling of pericoronary fat tissue around all three major epicardial coronary arteries in relation to CMR. Follow-up CCTA for the evaluation of changes in pericoronary fat attenuation will also be performed. MINOCA-GR aims to be the first study to explore the role of CCTA in combination with CMR and ICA in the underlying pathophysiological mechanisms and assisting in diagnostic evaluation and prognosis of patients with MINOCA.

Ethics and dissemination The study protocol has been approved by the institutional review board/ independent ethics committee at each site prior to study

\section{Strengths and limitations of this study}

- Myocardial infarction with non-obstructive coronary arteries (MINOCA) patients will undergo multimodality imaging with cardiac CT angiography (CCTA) in addition to cardiac MR (CMR).

- Use of CCTA to study atherosclerotic plaque burden and pericoronary fat attenuation in MINOCA patients.

- Enrolment based on invasive coronary angiogram and CMR.

- Vasomotor tests and invasive intracoronary imaging will be not routinely performed.

- MINOCA patients will be enrolled in a national registry beyond the target cohort size of 60 .

commencement. All patients will provide written informed consent. Results will be disseminated at national meetings and published in peer-reviewed journals.

Trial registration number NCT4186676.

\section{INTRODUCTION}

Myocardial infarction with non-obstructive coronary arteries (MINOCA) is characterised by clinical features of an acute myocardial infarction (AMI) but without evidence of obstructive coronary artery disease (CAD) on invasive coronary angiography (ICA). ${ }^{1}$ Diagnosis of the syndrome should be made immediately on ICA in patients presenting with features consistent with an AMI, as detailed by the following criteria: universal AMI criteria, ${ }^{2}$ non-obstructive coronary arteries on 
angiography (defined as no coronary artery stenosis $\geq 50 \%$ in any major epicardial coronary artery) and no clinically overt specific cause for the acute presentation. Moreover, non-ischaemic causes for myocyte injury (myocarditis or takotsubo cardiomyopathy) and alternative diagnoses (pulmonary embolism) should be excluded. ${ }^{3}$

MINOCA is reported in 5\%-15\% of all patients with AMI. Female sex and younger age are more frequently observed in patients with MINOCA compared with patients with AMI and obstructive CAD. ${ }^{3-5}$ Plaque rupture or ulceration, coronary spasm, thrombosis or thromboembolism, coronary dissection and ischaemic myocardial injury attributable to supply/demand mismatch have been proposed as potential pathophysiological mechanisms. ${ }^{6-8}$ MINOCA patients seem to have better outcomes compared with their AMI counterparts with obstructive CAD. However, they do carry a high risk for recurrent symptoms and events. ${ }^{9-11}$ Optimal management of MINOCA patients requires understanding of the underlying pathophysiological mechanism and there is lack of published randomised clinical trial regarding treatment. ${ }^{12}$ Furthermore, secondary preventive treatment of patients with AMI and obstructive CAD has not been validated in patients with MINOCA. Thus, evidence-based guidelines for treatment of MINOCA are lacking.

Recent multimodality imaging studies have revealed the utility of cardiac MR (CMR) and optical coherence tomography (OCT) to identify the underlying pathophysiological mechanism in MINOCA. ${ }^{78}$ The role of cardiac CT angiography (CCTA) in patients with MINOCA, however, has not been well studied so far. Apart from detecting coronary artery anomalies, stenosis and myocardial bridges ${ }^{13}$ CCTA has a suite of strengths in the identification of vulnerable plaque features and total atherosclerotic plaque burden. Importantly, CCTA can identify pericoronary fat attenuation and local inflammation, ${ }^{14}{ }^{15}$ the role of which has not been well studied in MINOCA. As a result, CCTA combined with CMR could assist with non-invasive identification of the pathophysiological mechanism, risk stratification and prognosis of MINOCA patients.

MINOCA-GR is a prospective study based on a national registry aiming to collect data regarding the epidemiology, symptomatology, cardiovascular risk factors, management and outcomes of patients with MINOCA in Greece. The study will particularly investigate the role of CCTA in combination with CMR and ICA in determining mechanisms of MINOCA and predicting outcomes.

\section{METHODS}

\section{Study design}

MINOCA-GR (NCT4186676) is an ongoing, prospective, multicentre, observational cohort study based on a national registry of patients presenting with MINOCA in Greece. The target cohort size is 60 consecutive and prospectively enrolled adult patients across four participating study sites within 12 months. The national registry
Box 1 Study inclusion and exclusion criteria

\section{Inclusion criteria}

- Patients older than 18 years without known history of coronary artery disease.

- Patients with acute coronary syndrome, with and without STsegment elevation, who underwent invasive coronary angiography within $24 \mathrm{~h}$ hours after onset of symptoms.

- Normal coronary arteries or plaques causing $<50 \%$ angiographic stenosis based on the results of invasive coronary angiography.

Exclusion criteria

- Patients with a history of obstructive coronary artery disease (eg, angiographic $\geq 50 \%$ diameter stenosis) and/or prior revascularisation.

- Myocarditis or/and takotsubo cardiomyopathy, based on cardiac MRI.

- Estimated glomerular filtration rate $<30 \mathrm{~mL} / \mathrm{min}$ or contraindication to additional contrast needed for cardiac CT angiography imaging.

Pregnancy and/or breast feeding.

will continue to enrol patients beyond 12 months. Diagnosis will be made on ICA and CMR in a patient presenting with features consistent with an AMI if the patient meets the following criteria: universal AMI criteria, ${ }^{2}$ nonobstructive coronary arteries on angiography (defined as no coronary artery stenosis $\geq 50 \%$ in a major epicardial coronary artery) and no clinically overt specific cause for the acute presentation. The study exclusion criteria are as follows: (1) age $<18$ years old at the time of coronary angiography; (2) known history of $\mathrm{CAD}$ and/or prior revascularisation; (3) diagnosis of myocarditis and/or takotsubo cardiomyopathy based on CMR; (4) serious concurrent disease and life expectancy of $<1$ year; (5) pregnancy and/or breast feeding; (6) estimated glomerular filtration rate $<30 \mathrm{~mL} / \mathrm{min}$; (7) known allergy to contrast agent that cannot be adequately premedicated (box 1). The primary goal of the study is to evaluate the coronary anatomy, characteristics of coronary atherosclerosis and pericoronary fat attenuation by CCTA, and study their correlation with CMR findings. CCTA will be performed at baseline and 6 months after index hospitalisation. Clinical outcomes will also be recorded as part of the registry. All subjects will provide written informed consent before study entry, at the time of ICA. The MINOCA-GR study is registered with www.clinicaltrials.gov. ${ }^{16}$

\section{Data collection}

ICA will be performed at the time of index hospitalisation. Subjects with angiographically normal coronary arteries or absence of obstructive coronary artery lesions (diameter stenosis $<50 \%$ ) will undergo screening for study eligibility. Operators in all participating sites are strongly encouraged to use intracoronary imaging, either intravascular ultrasound (IVUS) or OCT, of suspected culprit lesions. Data will be collected at baseline, 30 days, 6 months and 12 months after index hospitalisation as summarised in table 1. During index hospitalisation, CMR imaging will be performed in all patients for the evaluation of inflammation and scar. The CMR protocol will include imaging for cardiac function, late 
Table 1 Chart of the MINOCA-GR data collection and visit calendar

\begin{tabular}{|c|c|c|c|c|}
\hline Event & Index hospitalisation & 30 days & 6 months & 12 months \\
\hline Type of contact & In person & In person & In person & In person \\
\hline Inclusion/exclusion criteria & $\nabla$ & & & \\
\hline Physical examination & $\nabla$ & $\square$ & & $\nabla$ \\
\hline Demographics and clinical profile & $\nabla$ & & & \\
\hline Laboratory tests & $\nabla$ & $\nabla$ & & \\
\hline Invasive coronary angiography & $\nabla$ & & & \\
\hline Cardiac MR & $\nabla$ & & & \\
\hline Coronary CT angiography & $\nabla$ & & $\nabla$ & \\
\hline Electrocardiography & $\nabla$ & $\nabla$ & & $\nabla$ \\
\hline Anginal status (SAQ) & $\nabla$ & $\nabla$ & $\nabla$ & $\nabla$ \\
\hline Quality of life measurements & $\nabla$ & & & $\nabla$ \\
\hline
\end{tabular}

CAD, coronary artery disease; MINOCA, myocardial infarction with non-obstructive coronary arteries; SAQ, Seattle Angina Questionnaire.

gadolinium enhancement (LGE), and T1 mapping and/ or T2-weighted imaging for myocardial oedema. Following CMR and during index hospitalisation, all subjects will undergo CCTA for the quantification of total atherosclerotic plaque burden, characterisation of adverse plaque features, detection of coronary artery anomalies and myocardial bridges, and evaluation of local inflammation by pericoronary fat attenuation phenotyping (figure 1). Medical plan and clinical treatment strategy, pre-CCTA and post-CCTA,

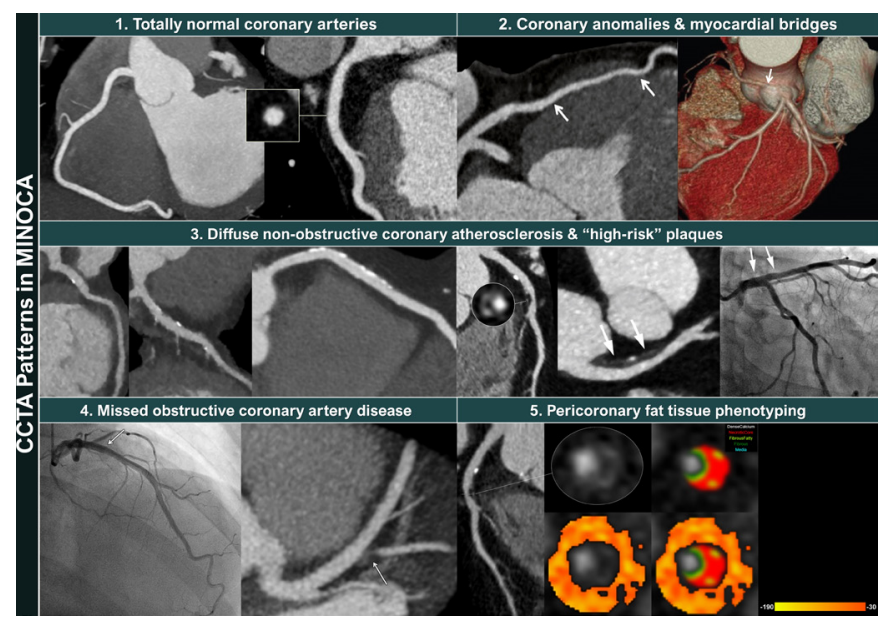

Figure 1 CCTA patterns in patients who initially diagnosed with MINOCA. (1) Totally normal coronary arteries, (2) coronary artery anomalies and myocardial bridges, (3) diffuse non-obstructive coronary atherosclerosis and 'high-risk' plaques, (4) missed obstructive coronary artery disease and (5) pericoronary fat attenuation profiling. CCTA, cardiac CT angiography; MINOCA, myocardial infarction with nonobstructive coronary arteries. will be documented separately. A repeat CCTA scan will be performed 6 months after the acute event to detect dynamic attenuation changes in pericoronary fat tissue around all three major epicardial coronary vessels.

\section{CCTA image acquisition}

CT scans will be performed using a 64-slice or 160slice scanner with prospective ECG triggering. CT protocol optimisations will be performed at all sites throughout the study, to minimise radiation dose and contrast exposure. One hour prior to CT scanning, patients with a heart rate of greater than 60 beats $/ \mathrm{min}$ and systolic blood pressure $>110 \mathrm{~mm} \mathrm{Hg}$ will receive oral metoprolol with staggered dosage based on the presenting resting heart rate, ranging from 50 to $100 \mathrm{mg}$, followed by supplemental intravenous doses immediately prior to the CT scan if the target heart rate $(<60 \mathrm{bpm})$ is not achieved. ${ }^{17} \mathrm{~A}$ small dose of oral diazepam may be prescribed for anxious patients, to improve heart rate control. Sublingual glyceryl trinitrate will be administered immediately prior to CT imaging. A non-enhanced ECG-synchronised scan for the detection and quantification of coronary calcium will be performed prior to coronary angiography. Coronary angiography will be conducted during contrast enhancement using bolus tracking protocol during a single breath-hold with prospective ECG triggering as appropriate.

\section{CCTA image analysis}

All CCT angiograms will be analysed independently by three experienced readers with level three training according to European Association of Cardiovascular 
Imaging/European Society of Cardiology (EACVI/ ESC) levels of competence ${ }^{18}$ who are blinded to all clinical information. A subsequent joint reading will be performed, and a consensus will be reached. The Agatston score will be calculated using semiautomated computerised software with a threshold of 130 Hounsfield units. ${ }^{19}$ The calcium score percentile based on age and sex will be calculated using coronary artery calcium score distributions from the Multi-Ethnic Study of Atherosclerosis. ${ }^{20}$ CCT datasets will be analysed using dedicated software for vessel analysis with tools for semiautomatic quantification of plaque volume (3mensio Structural Heart, Pie Medical Imaging BV, The Netherlands). For the grading of stenosis severity, a classification system suggested by the Society of Cardiovascular CT will be used. ${ }^{21}$ Moreover, high-risk plaque features (figure 2) and Coronary Artery Volume Index (CAVi, figure 3$)^{22}$ will be evaluated. Finally, total atherosclerotic plaque burden will be assessed on a perpatient basis using previous scoring systems such as Leiden CTA risk score,${ }^{23}$ CT-adapted Gensini score,${ }^{24}$ segment involvement score and segment severity score ${ }^{25}$ Pericoronary fat attenuation measurements will be carried out by a semiautomated analysis using PMOD V.3.805 (PMOD Technologies, Zurich, Switzerland), as previously reported. $^{15}$

\section{Infarct-related artery analysis}

For each patient, a feeding coronary artery will be assigned to each myocardial segment on the basis of the American Heart Association recommendations ${ }^{26}$ by independent investigators, blinded to the results of CMR. An infarct-related artery (IRA) on the basis of LGE distribution and myocardial oedema will then be identified. A subsequent analysis will be performed per segment for plaque quantification and high-risk plaque features (figure 2), and pericoronary fat attenuation profiling. Finally, differences between coronary plaques located in IRAs and those located in non-IRAs will be documented.

\section{Study endpoints}

The primary and secondary endpoints are summarised in box 2 and include both imaging and clinical variables. In terms of CCTA endpoints, quantification of the extent and severity of coronary atherosclerosis (figures 4 and 5), prevalence and classification of myocardial bridges, frequency and description of high-risk plaque features (figure 2), CAVi (figure 3) and pericoronary fat attenuation measurements will be recorded. Pericoronary fat attenuation in particular will be assessed during the index-hospitalisation and 6 months after. The MINOCA-GR registry will also include incidence (\%) of death and hospitalisation for major cardiovascular events during follow-up, frequency (\%) of pre-MI and post-MI angina, documentation of pre-CCTA and post-CCTA treatment

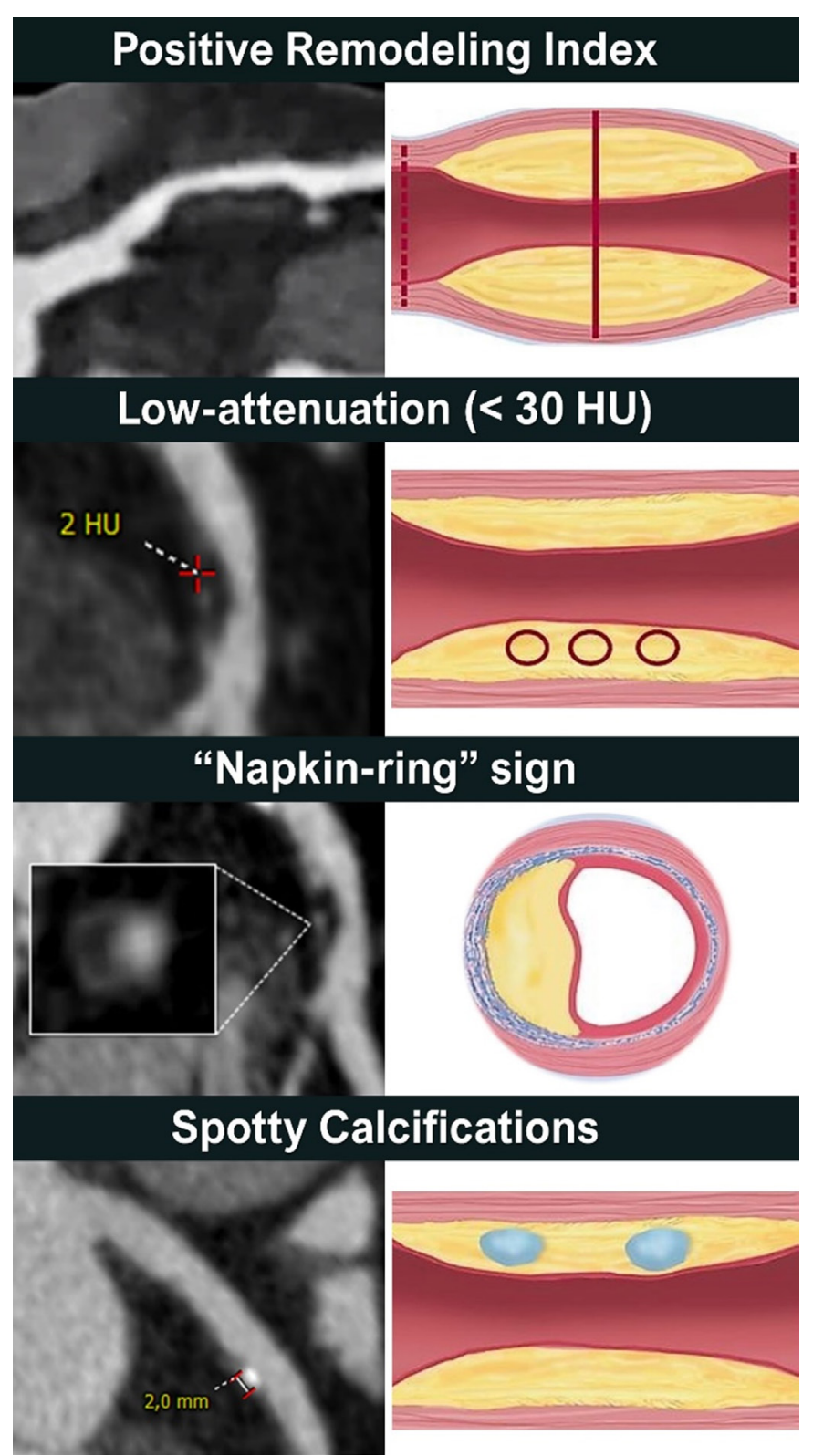

Figure 2 High-risk plaque features on coronary CTA. The analysis of ECG-synchronised coronary CTA images permits accurate assessment of both the presence and degree of luminal obstruction and the presence, morphology and composition of coronary atherosclerosis, including highrisk plaque features, such as positive remodelling, low CT attenuation plaque, 'napkin-ring' sign, and spotty calcium. CTA, CT angiography; HU, Hounsfield units.

strategy, assessment of quality of life. A nested registry of consecutive patients with AMI and obstructive CAD will be created from one of the participating sites.

\section{Follow-up}

Patients will be prospectively followed for 12 months after enrolment. Follow-up will include a phone contact at 6 months to record potential primary endpoints and a clinic visit at 1 and 12 months to reassess clinical, lab and imaging parameters and record primary and secondary endpoints. The estimated total duration of the study from first patient 


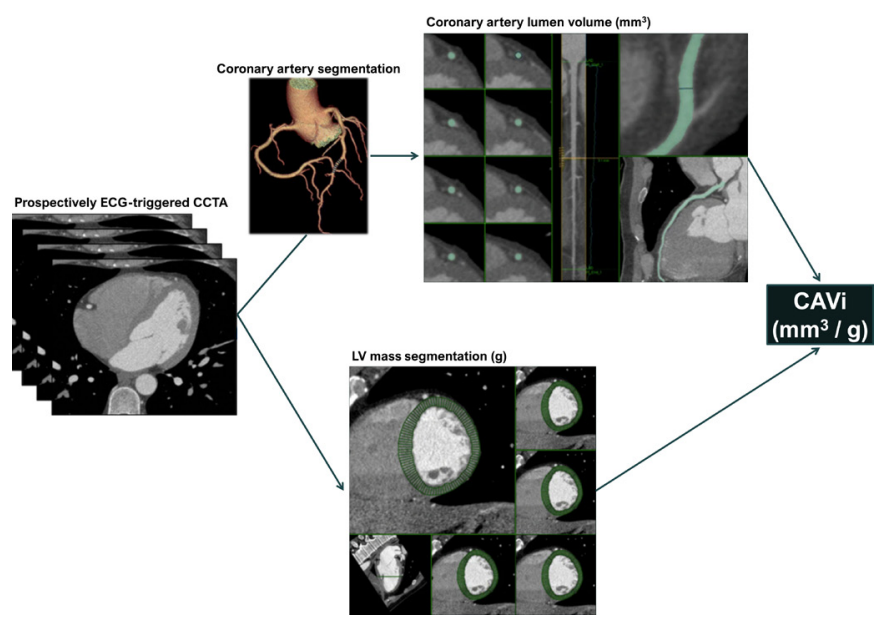

Figure 3 Methodology for computing Coronary Artery Volume index (CAVi). The coronary artery vessel tree is segmented from the CCTA dataset and the coronary artery volume (ie, lumen) is calculated for all vessels and branches $\geq 1.5 \mathrm{~mm}$ in diameter. LV myocardial mass is extracted from the CCTA dataset and computed with a dedicated software. Finally, CAVi is computed by dividing coronary artery volume over LV mass. CCTA, cardiac CT angiography; LV, left ventricle.

\section{Box 2 Myocardial infarction (MI) with non-obstructive} coronary arterie-GR study endpoints

\section{Primary endpoints}

1. Frequency (\%) of post-Ml angina as assessed by Seattle Angina Questionnaire (SAQ). (Time frame: 1, 6 and 12 months)

2. Extent of coronary atherosclerosis according to Leiden CT angiography (CTA) risk score, total atherosclerotic plaque volume $\left(\mathrm{mm}^{3}\right)$ and CT-adapted Gensini score. (Time frame: during index hospitalisation)

3. Frequency of occurrence (\%) of high-risk plaques according to cardiac CTA (CCTA). (Time frame: during index hospitalisation)

4. Prevalence of coronary anomalies and myocardial bridges as assessed with CCTA. (Time frame: during index hospitalisation)

5. Pericoronary fat attenuation index measured in each major epicardial coronary artery. (Time frame: during index hospitalisation and 6 months after the acute event)

\section{Secondary endpoints}

1. Incidence (\%) of composite events, including death, acute coronary syndrome, coronary revascularisation, heart failure decompensation, systemic or pulmonary embolism, intracardiac thrombosis, major bleeding or atrial fibrillation. (Time frame: 1, 6 and 12 months)

2. Generic health status and quality of life measurements. (Time frame: 12 months)

3. Frequency of pre-MI angina as assessed by SAQ. (Time frame: prior index-hospitalisation)

4. Frequency (\%) of chest pain rehospitalisation. (Time frame: 12 months)

5. Any type of rehospitalisation. (Time frame: 12 months)

6. Indexed Coronary Volume Index as assessed with CCTA. (Time frame: during index hospitalisation)

7. Documentation of pre-CCTA and post-CCTA treatment strategy. (Time frame: before and after CCTA examination)

8. Outpatient medication profile in real-life basis. (Time frame: At hospital discharge and 12 months after the index event) screened to last patient last visit will be 24 months. The MINOCA-GR study timeline is presented in figure 6 .

\section{Statistical analysis and sample size estimation}

Approximately 1000 patients are estimated to be hospitalised with AMI in the participating clinical sites over the enrolment period. Given that the known prevalence of MINOCA is estimated to be $6 \%$ among patients diagnosed with MI and considering a drop-out rate of $10 \%$, a cohort size of $\mathrm{N}=60$ patients is a realistic goal for such a clinical study in the Greek population. Statistical analyses will be descriptive, exploratory and generally limited to frequency tables or summary statistics. All values will be presented with a corresponding $95 \%$ CI and $p<0.05$ will be accepted as the level of significance. Continuous variables will be expressed as mean $\pm \mathrm{SD}$ or median and IQR; 25th, 75th percentile depending on normality of the distribution. Categorical values will be presented as frequencies and percentages (\%). Intervariable comparisons will be conducted, using analysis of variance, $\chi^{2}$ test or Fisher's exact tests where appropriate. Time-to-event endpoints will be plotted with the use of the KaplanMeier method, measured from the time of enrolment to the time of occurrence of the first event.

\section{Subject confidentiality}

Data will be centrally stored in a structured electronic database and will be only accessible by study staff. Strict subject confidentiality will be maintained through subject identification codes.

\section{Data and safety monitoring}

At multiple time points a data and safety monitoring board consisting of study investigators and an independent statistician will review accumulating data for quality and safety and will report back to the steering committee of the study.

\section{Patient and public involvement}

There has been no public or patient involvement in the design of this study. The study results will be disseminated to the participating patients via the investigators once they become published in a peer-reviewed journal.

\section{DISCUSSION}

MINOCA-GR is the first nationwide, prospective, observational study involving consecutive MINOCA patients who undergo ICA and multimodality imaging with CMR and CCTA during index-hospitalisation. CCTA will also be performed at follow-up to evaluate for changes in pericoronary fat attenuation and progression of atherosclerosis. Specifically, the study will evaluate the role of CCTA in combination with CMR in the evaluation of the underlying pathophysiological mechanisms in MINOCA patients. Despite the strengths and advantages of CCTA, it has not yet been incorporated in the evaluation of the underlying mechanisms of MINOCA. Moreover, MINOCA 

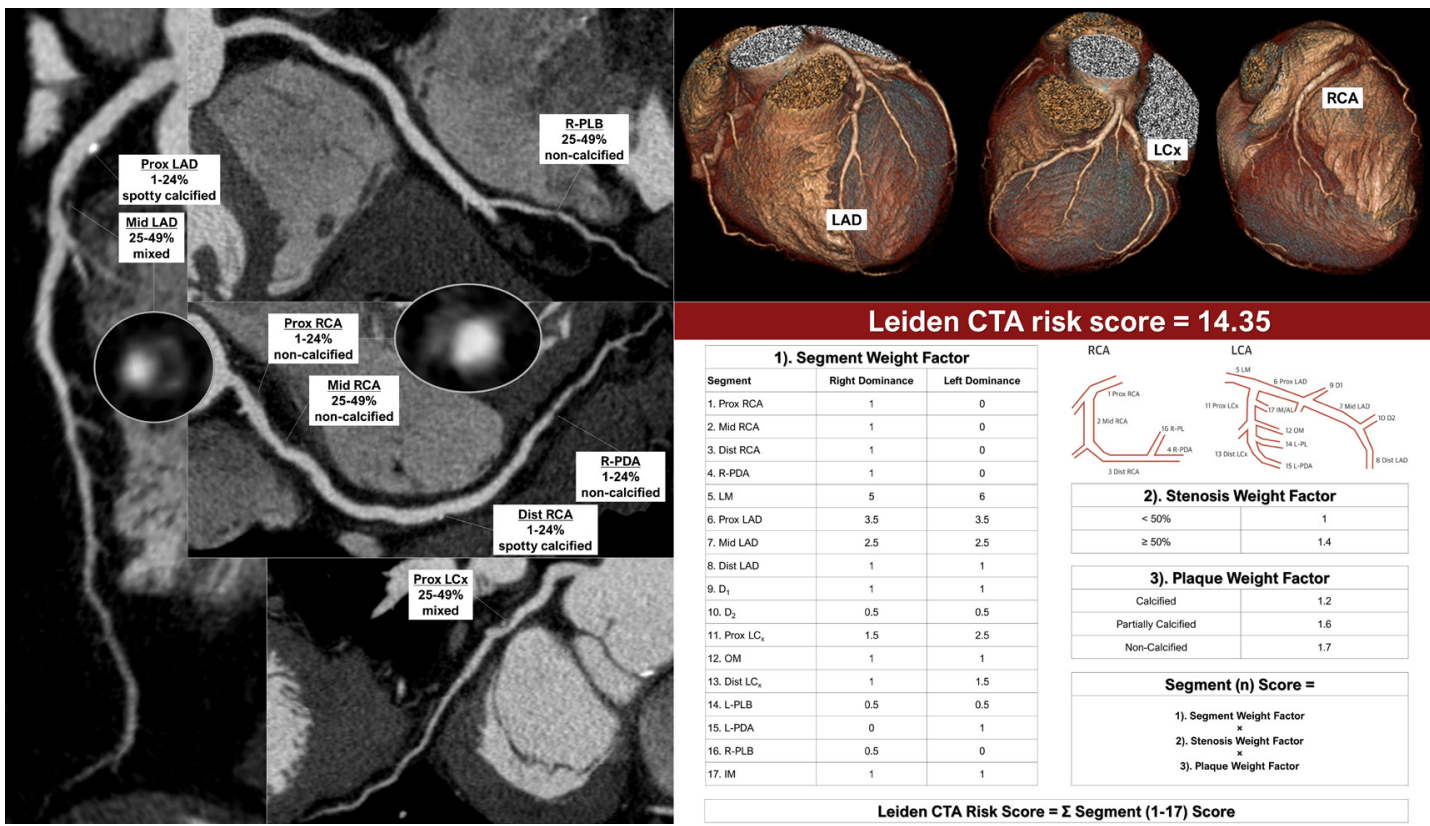

Figure 4 Leiden CTA risk score. An example of Leiden CTA risk score calculation. The new, comprehensive CTA score is calculated by addition of the individual segment scores, which are obtained by multiplication of the plaque weight factor, the stenosis weight factor, and the location weight factor. Leiden CTA risk score calculator is available at: http://18.224.14.19/ calcApp/. CTA, CT angiography; R-PDA, right posterior descending artery; RCA, right coronary artery; LCA, left coronary artery; LAD, left anterior descending; LCx, left circumflex; R-PLB, right posterolateral branch.

patients have not yet been systematically investigated in the Greek population. The study will collect realworld data regarding prevalence, demographics, medical history, medication profile, management and outcomes in MINOCA patients in Greece.

In patients with MINOCA, CMR imaging provides insights into potential causes while also excluding myocarditis and/or takotsubo cardiomyopathy. CMR assesses myocardial oedema, perfusion and tissue morphology. LGE is useful not only in localising the area of myocardial damage, but also in understanding whether the underlying mechanism is ischaemic or non-ischaemic. On the other hand, coronary artery characteristics identified by CCTA, such as content, volume and distribution of plaque, plaque characteristics, maximal luminal stenosis, and pericoronary inflammation could provide additional insights beyond CMR. Therefore, the combined use of both CMR and CCTA imaging may have a specific role in MINOCA.

Intravascular imaging modalities, such as IVUS and OCT, have been used as the gold standard to identify

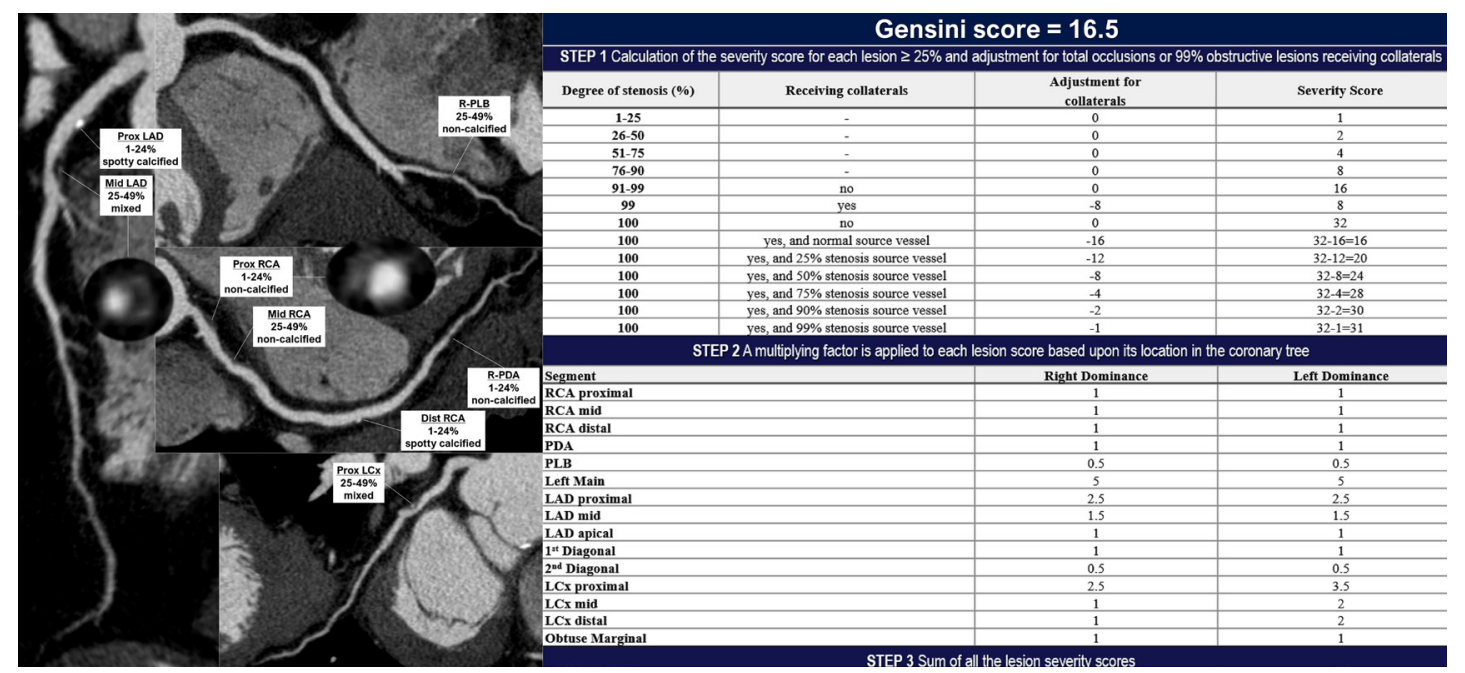

Figure 5 CT-adapted Gensini score calculation. An example of Gensini score calculation from CCTA dataset (curved multiplanar reconstruction) in a MINOCA patient. CCTA, cardiac CT angiography; MINOCA, myocardial infarction with nonobstructive coronary arteries; R-PDA, right posterior descending artery; RCA, right coronary artery; LCA, left coronary artery; LAD, left anterior descending; LCx, left circumflex; R-PLB, right posterolateral branch. 


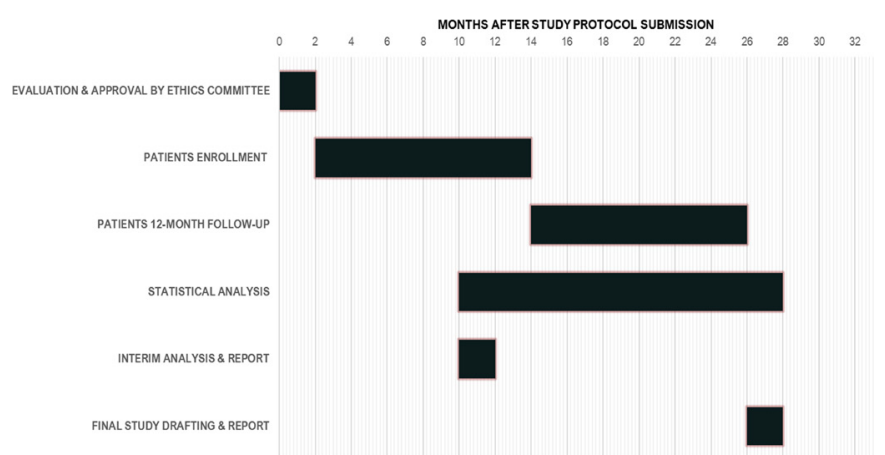

Figure 6 Timeline from protocol submission to study end.

plaque disruption, coronary dissection and thrombosis. They can identify the presence, extent, volume and characteristics of coronary atherosclerosis and have long contributed to help understand the mechanisms of AMI. ${ }^{27}{ }^{28}$ Vulnerable plaque features, which are predominantly associated with AMI, are commonly observed by IVUS or OCT. ${ }^{29}$ Specifically, IVUS is helpful in the early detection of remodelling progression, plaque rupture or erosion and coronary plaque stability. OCT appears to be more sensitive than IVUS in detecting ruptured plaques and thrombus, while also overcoming the limitations of IVUS in detecting plaque rupture, thrombus and intramural haematoma. ${ }^{30}$ However, both are invasive modalities that may be impractical to perform routinely in multiple vessels at the time of AMI.

CCTA has been widely adopted due to its high diagnostic yield, becoming a key imaging modality in patients with stable chest pain. ${ }^{31}{ }^{32}$ Even though detection of stenosis severity and plaque volume was the initial object of interest in previous CCTA studies, more recently, the detection of high-risk plaques gained interest. CCTA is capable of identifying highrisk plaque features with comparable results to intracoronary imaging modalities for this purpose. ${ }^{33-35}$ CCTA is also excellent in monitoring plaque development, including progression, regression or stabilisation, appearing non-inferior to IVUS. ${ }^{36}$ The ability of CCTA to evaluate the entire coronary vessel for atherosclerosis (as compared with only segmental approach by IVUS or OCT) is crucial to identify and predict ischaemia. ${ }^{37-39}$ In addition, CCTA can now be used for the evaluation of pericoronary fat tissue with valuable prognostic implications. ${ }^{14} 1540$ Recent advances in artificial intelligence and deep learning ${ }^{41}$ may result in a reduction of radiation dose during CCTA.

The MINOCA-GR study will explore the role of CCTA in combination with CMR and ICA in the identification of the underlying mechanism and potentially in prognostication of patients with MINOCA. It will also provide useful data regarding demographics, clinical characteristics, management and prognosis of patients with MINOCA in Greece.

\section{ETHICS AND DISSEMINATION}

This study will be conducted in keeping with the International Conference on Harmonisation Good Clinical Practice guidelines and the Declaration of Helsinki. All participating sites have obtained approval from appropriate independent ethics committees or institutional review boards. The principal investigator will promptly report to the ethics committees all changes in research activity and all unanticipated problems involving risks to human subjects or others and will not make any changes in the protocol without approval by the responsible ethics committees. Each patient must sign and date the approved informed consent form after the study procedures have been fully explained.

\section{Author affiliations}

${ }^{1}$ First Department of Cardiology, University General Hospital of Thessaloniki AHEPA, Thessaloniki, Greece

${ }^{2}$ Cardiac Imaging Unit, Diagnostic Center "PANAGIA", Veroia, Greece

${ }^{3}$ Division of Cardiology, NYU Langone Medical Center, New York, New York, USA

${ }^{4}$ Department of Radiology, University General Hospital of Thessaloniki AHEPA,

Thessaloniki, Greece

${ }^{5}$ First Department of Cardiology, Hippokration Hospital, Athens, Greece

${ }^{6}$ Department of Nuclear Medicine - Cardiac Imaging Unit, University Hospital Zurich, Zurich, Switzerland

${ }^{7}$ Radiology Department, St Luke's Hospital, Thessaloniki, Greece

${ }^{8}$ Department of Cardiology, General Hospital of Veroia, Veroia, Greece

${ }^{9}$ Sarah Ross Soter Center for Women's Cardiovascular Research, Leon H. Charney Division of Cardiology, Department of Medicine, NYU Grossman School of Medicine, New York, New York, USA

\section{Twitter Nathaniel R Smilowitz @NSmilowitzMD}

Acknowledgements We thank all the patients, investigators, and site staff for participating in this study.

Contributors GPR and PNK: formal analysis; investigation; methodology; project administration; resources; supervision; validation; visualisation; roles/writingoriginal draft; writing-review and editing. KK, GB, AAG: methodology; project administration; resources; supervision; writing-review and editing. AS and AK: investigation; resources; writing-review. TK, AS, IV, SH, AZ, RRB, CG, NRS, KTo, KTs, PP and HK: investigation; resources; supervision; writing-review and editing. HR: investigation; methodology; resources; supervision; validation; visualisation; writing-review and editing. GG: conceptualisation; formal analysis; investigation; methodology; project administration; resources; supervision; validation; visualisation; roles/writing-original draft; writing-review and editing.

Funding MINOCA-GR is an investigator-initiated study, supported by Menarini Hellas S.A (Ref. No. 27.11.2020/72059).

Disclaimer The funder had no role in the design or conduct of the study; preparation, review, or approval of the manuscript; and decision to submit the manuscript for publication.

\section{Competing interests None declared.}

Patient and public involvement Patients and/or the public were not involved in the design, or conduct, or reporting, or dissemination plans of this research.

Patient consent for publication Not applicable.

Provenance and peer review Not commissioned; externally peer reviewed.

Open access This is an open access article distributed in accordance with the Creative Commons Attribution Non Commercial (CC BY-NC 4.0) license, which permits others to distribute, remix, adapt, build upon this work non-commercially, and license their derivative works on different terms, provided the original work is properly cited, appropriate credit is given, any changes made indicated, and the use is non-commercial. See: http://creativecommons.org/licenses/by-nc/4.0/.

\section{ORCID iDs}

Georgios P Rampidis http://orcid.org/0000-0002-2452-6942

loannis Vogiatzis http://orcid.org/0000-0002-6269-0292 
George Giannakoulas http://orcid.org/0000-0001-7491-6319

\section{REFERENCES}

1 Agewall S, Beltrame JF, Reynolds HR, et al. ESC Working group position paper on myocardial infarction with non-obstructive coronary arteries. Eur Heart J 2017;38:143-53.

2 Thygesen K, Alpert JS, Jaffe AS, et al. Fourth universal definition of myocardial infarction (2018). J Am Coll Cardiol 2018;72:2231-64.

3 Tamis-Holland JE, Jneid H, Reynolds HR, et al. Contemporary diagnosis and management of patients with myocardial infarction in the absence of obstructive coronary artery disease: a scientific statement from the American heart association. Circulation 2019;139:e891-908.

4 Haider A, Bengs S, Luu J, et al. Sex and gender in cardiovascular medicine: presentation and outcomes of acute coronary syndrome. Eur Heart J 2020;41:1328-36.

5 Smilowitz NR, Mahajan AM, Roe MT, et al. Mortality of myocardial infarction by sex, age, and obstructive coronary artery disease status in the action Registry-GWTG (acute coronary treatment and intervention outcomes network Registry-Get with the guidelines). Circ Cardiovasc Qual Outcomes 2017;10:e003443.

6 Scalone G, Niccoli G, Crea F. Editor's Choice- pathophysiology, diagnosis and management of MINOCA: an update. Eur Heart $J$ Acute Cardiovasc Care 2019;8:54-62.

7 Reynolds HR, Maehara A, Kwong RY. Coronary optical coherence tomography and cardiac magnetic resonance imaging to determine underlying causes of MINOCA in women. Circulation 2021;143:624-40.

8 Reynolds HR. Searching for underlying causes of MINOCA with multi-modality imaging. JACC Cardiovasc Imaging 2020;13:2632-4.

9 Choo EH, Chang K, Lee KY, et al. Prognosis and predictors of mortality in patients suffering myocardial infarction with nonobstructive coronary arteries. J Am Heart Assoc 2019;8:e011990.

10 Grodzinsky A, Arnold SV, Gosch K, et al. Angina frequency after acute myocardial infarction in patients without obstructive coronary artery disease. Eur Heart J Qual Care Clin Outcomes 2015;1:92-9.

11 Lindahl B, Baron T, Erlinge D, et al. Medical therapy for secondary prevention and long-term outcome in patients with myocardial infarction with nonobstructive coronary artery disease. Circulation 2017;135:1481-9.

12 Nordenskjöld AM, Agewall S, Atar D, et al. Randomized evaluation of beta blocker and ACE-inhibitor/angiotensin receptor blocker treatment in patients with myocardial infarction with non-obstructive coronary arteries (MINOCA-BAT): rationale and design. Am Heart J 2021;231:96-104.

13 Brolin EB, Brismar TB, Collste O, et al. Prevalence of myocardial bridging in patients with myocardial infarction and Nonobstructed coronary arteries. Am J Cardiol 2015;116:1833-9.

14 Oikonomou EK, Marwan M, Desai MY, et al. Non-Invasive detection of coronary inflammation using computed tomography and prediction of residual cardiovascular risk (the CRISP CT study): a post-hoc analysis of prospective outcome data. Lancet 2018;392:929-39.

15 Bengs S, Haider A, Warnock Gl, et al. Quantification of perivascular inflammation does not provide incremental prognostic value over myocardial perfusion imaging and calcium scoring. Eur J Nucl Med Mol Imaging 2021;48:1806-12.

16 Myocardial infarction with non-obstructive coronary arteries in the Greek population (MINOCA-GR). Available: https://clinicaltrials.gov/ ct2/show/NCT04186676

17 Abbara S, Blanke P, Maroules CD, et al. SCCT guidelines for the performance and acquisition of coronary computed tomographic angiography: a report of the Society of cardiovascular computed tomography guidelines Committee: endorsed by the North American Society for cardiovascular imaging (NASCI). J Cardiovasc Comput Tomogr 2016;10:435-49.

18 Pontone G, Moharem-Elgamal S, Maurovich-Horvat P, et al. Training in cardiac computed tomography: EACVI certification process. Eur Heart J Cardiovasc Imaging 2018;19:123-6.

19 Hecht HS, Blaha MJ, Kazerooni EA, et al. CAC-DRS: coronary artery calcium data and reporting system. An expert consensus document of the Society of cardiovascular computed tomography (SCCT). J Cardiovasc Comput Tomogr 2018;12:185-91.

20 McClelland RL, Chung H, Detrano R, et al. Distribution of coronary artery calcium by race, gender, and age: results from the multi-ethnic study of atherosclerosis (MESA). Circulation 2006;113:30-7.
21 Leipsic J, Abbara S, Achenbach S, et al. SCCT guidelines for the interpretation and reporting of coronary CT angiography: a report of the Society of cardiovascular computed tomography guidelines Committee. J Cardiovasc Comput Tomogr 2014;8:342-58.

22 Benetos G, Buechel RR, Gonçalves M, et al. Coronary artery volume index: a novel CCTA-derived predictor for cardiovascular events. Int $J$ Cardiovasc Imaging 2020;36:713-22.

23 van Rosendael AR, Shaw LJ, Xie JX, et al. Superior risk stratification with coronary computed tomography angiography using a comprehensive atherosclerotic risk score. JACC Cardiovasc Imaging 2019;12:1987-97.

24 Rampidis GP, Benetos G, Benz DC, et al. A guide for Gensini score calculation. Atherosclerosis 2019;287:181-3.

25 Pagali SR, Madaj P, Gupta M, et al. Interobserver variations of plaque severity score and segment stenosis score in coronary arteries using 64 slice multidetector computed tomography: a substudy of the accuracy trial. J Cardiovasc Comput Tomogr 2010;4:312-8.

26 Cerqueira MD, Weissman NJ, Dilsizian V, et al. Standardized myocardial segmentation and nomenclature for tomographic imaging of the heart. A statement for healthcare professionals from the cardiac imaging Committee of the Council on clinical cardiology of the American heart association. Circulation 2002;105:539-42.

27 Toutouzas K, Benetos G, Karanasos A, et al. Vulnerable plaque imaging: updates on new pathobiological mechanisms. Eur Heart $J$ 2015;36:3147-54.

28 Toutouzas K, Chatzizisis YS, Riga M, et al. Accurate and reproducible reconstruction of coronary arteries and endothelial shear stress calculation using 3D OCT: comparative study to 3D IVUS and 3D QCA. Atherosclerosis 2015;240:510-9.

29 Toutouzas K, Karanasos A, Tousoulis D. Optical coherence tomography for the detection of the vulnerable plaque. Eur Cardiol 2016;11:90-5.

30 Johnson TW, Räber L, di Mario C, et al. Clinical use of intracoronary imaging. Part 2: acute coronary syndromes, ambiguous coronary angiography findings, and guiding interventional decision-making: an expert consensus document of the European association of percutaneous cardiovascular interventions. Eur Heart $J$ 2019;40:2566-84.

31 Poon M, Lesser JR, Biga C, et al. Current evidence and recommendations for coronary cta first in evaluation of stable coronary artery disease. J Am Coll Cardiol 2020;76:1358-62.

32 Abdelrahman KM, Chen MY, Dey AK, et al. Coronary computed tomography angiography from clinical uses to emerging technologies: JACC state-of-the-art review. J Am Coll Cardiol 2020;76:1226-43.

33 Marwan M, Taher MA, El Meniawy K, et al. In vivo CT detection of lipid-rich coronary artery atherosclerotic plaques using quantitative histogram analysis: a head to head comparison with IVUS. Atherosclerosis 2011;215:110-5.

34 Tomizawa N, Yamamoto K, Inoh S, et al. Accuracy of computed tomography angiography to identify thin-cap fibroatheroma detected by optical coherence tomography. J Cardiovasc Comput Tomogr 2017;11:129-34.

35 Yang DH, Kang S-J, Koo HJ, et al. Coronary CT angiography characteristics of OCT-defined thin-cap fibroatheroma: a section-tosection comparison study. Eur Radiol 2018;28:833-43.

36 Nakanishi R, Alani A, Matsumoto S, et al. Changes in coronary plaque volume: comparison of serial measurements on intravascular ultrasound and coronary computed tomographic angiography. Tex Heart Inst J 2018;45:84-91.

37 Villines TC, Rodriguez Lozano P. Transitioning from stenosis to plaque burden in the cardiac CT era: the changing risk paradigm. $J$ Am Coll Cardiol 2020;76:2814-6.

38 von Knebel Doeberitz PL, De Cecco CN, Schoepf UJ, et al. Coronary CT angiography-derived plaque quantification with artificial intelligence CT fractional flow reserve for the identification of lesionspecific ischemia. Eur Radiol 2019;29:2378-87.

39 Dey D, Gaur S, Ovrehus KA, et al. Integrated prediction of lesionspecific ischaemia from quantitative coronary CT angiography using machine learning: a multicentre study. Eur Radiol 2018;28:2655-64.

40 Gaibazzi N, Martini C, Botti A, et al. Coronary inflammation by computed tomography Pericoronary fat attenuation in MINOCA and Tako-Tsubo syndrome. J Am Heart Assoc 2019;8:e013235.

41 Benz DC, Benetos G, Rampidis G, et al. Validation of deeplearning image reconstruction for coronary computed tomography angiography: impact on noise, image quality and diagnostic accuracy. J Cardiovasc Comput Tomogr 2020;14:444-51. 